\title{
Des ,Roten Krebses‘ Abschiedsmahnung Gelehrsamkeit, Ironie und Systemkritik auf einem Brünner Grabepitaph des 17. Jahrhunderts
}

\section{Boris Blahak}

The article deals with a hitherto unnoticed German literary monument in Brno located in the Gothic hall church St. James: the grave epitaph of the councilman Heinrich Nass (1649-1698), once owner of the Red Cancer pharmacy. Apart from the reconstruction of the damaged rhymed inscription the paper undertakes a linguistic and genre-related classification, puts the imagery and argumentation into relation to common stylistic features of German Baroque poetry and attempts an interpretation of the anonymous verses characterized by bourgeois erudition, pointed irony and late Baroque world theatre. Particular attention is paid to the function of meter change, code switching and ambiguous assignments between narrator and text. Finally didactic suggestions about the use of the grave epitaph with classes studying German as a foreign language at (Brno) university are included.

Brno - St. James - grave inscriptions - Baroque poetry - occasional poem - literature didactics - extra-universitary areas of learning

Der Beitrag beschäftigt sich mit einem bisher unbeachteten literarischen Denkmal des Deutschen in Brünn, das sich in der gotischen Hallenkirche St. Jakob befindet: das Grabepitaph des Ratsherrn Heinrich Nass (1649-1698), zu Lebzeiten Inhaber der Apotheke Zum roten Krebsen. Neben der Rekonstruktion der beschädigten gereimten Inschrift nimmt der Beitrag eine sprachliche und gattungsbezogene Einordnung vor, setzt die Bildsprache und Argumentationsmuster des Textes in Bezug zu verbreiteten Stilmerkmalen deutschsprachiger Barocklyrik und versucht eine Interpretation der von bürgerlicher Gelehrsamkeit, pointierter Ironie und spätbarockem Welttheater geprägten anonymen Verse. Besondere Aufmerksamkeit wird dabei der Funktion des Metrumwechsels, des Codeswitchings sowie doppeldeutiger Sprecher/Text-Zuordnungen geschenkt. Abschließend erfolgen didaktische Überlegungen zur Verwendung des Grabepitaphs im Rahmen des (Brünner) Hochschulunterrichts Deutsch als Fremdsprache.

Brno - St. Jakob - Grabinschriften - Barocklyrik - Gelegenheitsgedicht - Literaturdidaktik - außerschulische Lernräume

\section{Literarische Denkmäler des Deutschen im tschechischen öffentlichen Raum: die Textsorte ,Grabinschrift'}

Literarische Denkmäler des Deutschen, zumal aus der frühneuhochdeutschen Epoche, sind in den ehemaligen böhmischen Ländern im öffentlichen Raum nur noch selten erhalten. In Brünn (Brno), der historischen Hauptstadt Mährens, findet sich gleichwohl noch eine Anzahl von ihnen. Zumeist unbeachtet bzw. unveröffentlicht und nicht beschrieben, harren sie ihrer sprach- und literaturwissenschaftlichen Erfassung. In diesem Zusammenhang erweist sich die gotische St. Jakobskirche (kostel svatého Jakuba) in der Brünner Altstadt als wahrer Konzentrationspunkt. 
Die Gründe hierfür liegen zum einen darin, dass das Gotteshaus Anfang des 13. Jahrhunderts hauptsächlich für die deutsche Bevölkerung der Stadt errichtet wurde, die damals im Bereich der heutigen Běhounská-Straße siedelte (vgl. Dostál 1928: 54), zum anderen darin, dass andere im Mittelalter errichtete Kirchen nicht mehr existieren oder durch starke Beschädigung und folgende Erneuerung ihre ehemalige Innenausstattung verloren. ${ }^{1}$ In diesem ältesten erhaltenen Sakralbau der Altstadt haben sich literarische Monumente der ehemaligen deutschen Gemeinde erhalten, und zwar in Form gereimter Grabinschriften des späten 17. und frühen 18. Jahrhunderts, mithin des Barock-Zeitalters.

Als Textsorte stellt die Grabinschrift (auch ,Epitaph') eine Sonderform des Epigramms dar: einen Gebrauchstext, ein Gelegenheitsgedicht in zweckgebundener Redesituation (vgl. Segebrecht 1987: 130). Seit dem Erscheinen von Christian Hoffman von Hoffmannswaldaus Hundert Grab-Schrifften (1662) bildete sie sich als Subkategorie barocker Kurzlyrik heraus (vgl. Kühlmann 1982: 168). Innerhalb der Funeral- und Totenliteratur, dem weitaus größten Teil des überlieferten BarockSchrifttums, bilden poetische Grabinschriften zwar nur eine verhältnismäßig kleine Gruppe, dennoch machen sie den Geist der Epoche auf besonders authentische Weise erfahrbar: In ihnen liegt eine gesellschaftlich verbreitete Textsorte vor, in der sich nicht nur fast alle bedeutenden Dichter des 17. Jahrhunderts versucht haben; vielmehr dürften damals die meisten der Schrift Kundigen als Rezipienten wie Verfasser von Gedichten zu Gelegenheiten des menschlichen Lebens, besonders zu Todesfällen, ${ }^{2}$ praktischen Umgang mit Literatur gehabt haben (vgl. Segebrecht 1981: $4,14)$. So handelt es sich bei diesen ,Casualcarmina' um universelle, gewissermaßen allmenschliche und damit auch exemplarische lyrische Texte. In ihrer rhetorischen Zweckgerichtetheit, Anlassgebundenheit und Adressatenorientierung, durch ihr Eingebunden-Sein in das gesellschaftliche Leben (vgl. Segebrecht 1978: 431), mit ihrem ,öffentlichen' Charakter in Kirche, Friedhof, Kreuzgang oder Gruft, in ihrer repräsentativen Funktion als Statussymbol und aufgrund ihres hehren spirituellen Gegenstandes treffen sie nicht zuletzt Aussagen über die Bedeutung der Poesie im Angesicht des Todes: nämlich ihre Möglichkeit, die irdische Vergänglichkeit (zumindest für einen Moment) aufzuheben und auf Erden Ewigkeit zu stiften (vgl. Segebrecht 1981: 15).

Der vorliegende Aufsatz möchte einen Beitrag zur Dokumentation und Erschließung dieser Textsorte in den böhmischen Ländern leisten. Hierzu soll

1 St. Nikolaus (kostel svatého Mikuláše), eine Filialkirche von St. Jakob, wurde 1869-1870 zugunsten der Neugestaltung des ,Großen Platzes', heute Freiheitsplatz (Náměstí svobody), abgerissen. Die Kathedrale St. Peter und Paul (katedrála svatého Petra a Pavla), um 1180 als romanisches Bauwerk gegründet und im 14. Jahrhundert zur gotischen Basilika umgebaut, brannte 1643/45 während der Belagerung Brünns durch die Schweden ab, wurde 1651/52 notdürftig wiederhergestellt und erst 1743-1746 und 1777 im Inneren neu gestaltet (vgl. Dostál 1928: 54).

$2 \mathrm{Zu}$ vanitas (Vergänglichkeit) und memento mori (Gedenke des Todes!), den Schlagwörtern des Barockzeitalters s. bes. Ingen (1966: 115-119). 
exemplarisch ein bisher noch unerschlossener Text rekonstruiert, sprachlich und gattungsbezogen eingeordnet und inhaltlich analysiert werden, bevor abschließend didaktische Überlegungen zu seiner Verwendbarkeit im Literaturunterricht Deutsch als Fremdsprache im Rahmen der (Brünner) Hochschulgermanistik erfolgen.

\section{Die poetischen Grabinschriften von St. Jakob (Brünn)}

Ein Epitaph fällt in St. Jakob bereits aufgrund seiner aufwändigen künstlerischen Gestaltung und exponierten Platzierung ins Auge: Hinter dem Hauptaltar ,entfaltet sich' das geradezu theatralisch inszenierte Grabmal des kaiserlichen Feldmarschalls Louis Rattuit de Souches (1608-1683): Der Feldherr kniet als Bronzestatue geharnischt mit der Geste eines Oranten über seinen militärischen Insignien auf seinem Sarkophag (Abb. 1). Die beigefügten exorbitanten 50 Alexandriner-Verse in deutscher Sprache sind aufgrund ihrer panegyrischen Darstellung der Tugenden und militärischen Leistungen des Verstorbenen unschwer der Textgattung des Fürstenslobs zuzuordnen. ${ }^{1}$

Eine weitere Grabinschrift, die im Folgenden eingehender betrachtet werden soll, steht diesem Fürstenepitaph in vielerlei Hinsicht (Stand des Verstorbenen, Platzierung, Aufwand der Gestaltung, Textumfang, Versmaß, Sprache und Inhalt) beinahe diametral gegenüber und macht gerade dadurch den durch Spannung und Gegensatz bestimmten Geist der Epoche des literarischen Barock greifbar. Schon seine periphere Positionierung, seine bescheidene bildhauerische Bearbeitung und nicht zuletzt sein defizitärer Erhaltungszustand unterscheiden das betreffende Grabmal grundlegend von jenem des Grafen de Souches: Platziert in der schwach beleuchteten Ecke zwischen dem nördlichen Seitenschiff und der Westfront von St. Jakob, findet sich ein von starker Verwitterung und durch drei diagonal verlaufende Bruchstellen gezeichneter äußerlich unspektakulärer Grabstein aus weißem Sandstein (Abb. 2 und 3). ${ }^{2}$

Die einschlägigen biographischen Angaben, die das Epitaph zwischen acht paarweise gereimten Versen und einer abschließenden Vers-Triole preisgibt, weisen den Verstorbenen gleichermaßen als ,internationale wie ,lokale Persönlichkeit aus: „NEMBLICH | DER WOHL EDLE VNDT GESTRENGE | HERR HENRİCVS NASS | RATHS VERWANDTER VNDT | APOTEKER ZVM ROTHEN KREBSEN VON | MAINTZ GEBVRTIG SEINES ALTERS 49 | İAHR STARBE DEN 22. IVLŸ ANNO 1698“. Bei dem Verstorbenen handelte es sich demnach um einen vom Rhein Zugezogenen, dem es (als Apotheker und Ratsherrn) in kurzer Zeit gelungen war, nicht nur ein voll anerkanntes, sondern sogar tonangebendes

1 Zur Textedition und -interpretation sowie zum Einsatz des Epitaphs im Literaturunterricht Deutsch als Fremdsprache s. Blahak (2005).

2 Die bisher einzige photographische Reproduktion in der Sekundärliteratur findet sich bei Bretholz (1901: 140), allerdings ohne Transkription des Textes und ohne inhaltlichen Kommentar. 
Mitglied der Brünner Bürgerschaft, des städtischen Patriziat, das die Spitze der sozialen Pyramide bildete, zu werden. Die Apotheke Zum roten Krebsen, die er neben seinem öffentlichen Amt führte, gibt es unter dem Namen Lékárna v domě $U$ červeného raka (Masarykova 1) in Brünn noch heute (Abb. 4).

\section{Das ,unspektakuläre‘ Grabepitaph des Brünner Ratsherrn Heinrich Nass}

\subsection{Textrekonstruktion, Sprache und Autorschaft}

Der v. a. durch die genannten Bruchstellen und deren grobes Verkitten nur fragmentarisch entzifferbare lyrische Teil der Grabinschrift entpuppt sich als poetisch-gelehrter, pointierter letzter Ratschlag eines Dahingeschiedenen an den Brünner Rat der Stadt, dem er selbst zu Lebzeiten angehört hatte. Der lesbare Text stellt sich zunächst wie folgt dar: ${ }^{1}$

(1)

$$
\begin{gathered}
\text { [...] RATHAVS AVFRİCHTİGEN } \\
\text { RATH ZV ERTHEİLEN } \\
\text { DER HİER RVHT VÖRLASE } \\
\text { ZWO FOLGENDE ZEILEN } \\
\text { HÆC DOMVS ODİT, AMAT, PVNIT, } \\
\text { CONSERVAT, HONORAT, } \\
\text { NEqVİTİAM, PACEM, CRİMİNA, } \\
\text { İVRA, PROBAT } \\
\text { DİS HAVS HAST, LİEBT, STR[...] } \\
\text { BEHALTET, VERE[...]ET } \\
\text { DİE SCHA[...]KHEIT [...]D, LASTER, } \\
\text { RECHT [...]T. DİS HERET, } \\
\text { RATHS H[...]RN: DEN REIM, NİE } \\
\text { LAST [...]S DEM SİN KOMMEN } \\
\text { SO HİNDERT İHR BESE VNDT } \\
\text { MEHRET DİE FROMMEN, } \\
\text { LESER NACH SEİNEM TODT, } \\
\text { HİLF İHM AVS SEINER NOTH } \\
\text { SPRICH: GNADİG SEİ İHM GOTT. }
\end{gathered}
$$

Da sich die Zeilen (9)-(12) als deutsche Übertragung der auf Latein verfassten Zeilen (5)-(8) erweisen, lässt sich der Textverlust an den meisten Stellen zweifelsfrei rekonstruieren: So ergibt sich für lat. „PVNIT“ (5) dt. „STR[AFET]“ (9), für lat. „HONORAT“ (6) dt. „VERE[HR]ET“ (10), für lat. „NEqVITIIAM“ (Akk., 7) dt. „SCHA[LC]KHEIT“ (11) und für lat. „PACEM“ (Akk., 7) dt. „[FRİ]D“ (11).

1 Die auf dem Epitaph vorgefundene Zeileneinteilung wird im Folgenden in runden Klammern beziffert. 
Andere fehlende Textteile lassen sich mithilfe erhaltener Wortbestandteile und des Textzusammenhangs ergänzen: so der bestimmte Artikel „[DEM]“ (1), ferner das Plural-Substantiv „H[ER]RN“ (13) und die Präposition „,[AV]S“ (14). Neben der Länge der jeweiligen Textlücke gab hier z. T. auch das Vers-Metrum, auf das im Folgenden noch eingegangen werden soll, entscheidende Hinweise.

Gewisse Schwierigkeiten bereitete lediglich die Textlücke in Zeile (12), in der ein Verb von ca. sechs Buchstaben Länge fast vollständig fehlte; aufgrund des in den Zeilen (1)-(4) und (9)-(16) strikt durchgehaltenen Amphibrachys ( $u-u$ ) stand immerhin fest, dass hier ein Äquivalent des korrespondierenden lateinischen „PROBAT“ (4) von zwei Silben Länge mit Erstsilbenbetonung (3. Person Präsens Indikativ) zu ergänzen war. Da ein- (prüft), drei- oder mehrsilbige Varianten (genehmigt, untersucht, beurteilt, beglaubigt) bzw. zweisilbige Varianten mit Zweitsilbenbetonung (be-lerweist, erprobt) aufgrund der metrischen Vorgaben ausschieden und trennbare Verben (stimmt zu, erkennt an, heißt gut, legt dar, lässt gelten, weist nach) oder Funktionsverbgefüge (schenkt Beifall) zusätzlich aufgrund der Satzstellung nicht in Frage kamen, scheint billigt, verschriftlicht als «BİL̇GT, die plausibelste Rekonstruktion des Textverlustes darzustellen.

Somit lautet die wieder vollständig lesbar gemachte Grabinschrift in Verseinteilung: ${ }^{1}$

[DEM] RATHAVS AVFRİCHTIGGEN | RATH ZV ERTHEILEN |

DER HİER RVHT VÖRLASE | ZWO FOLGENDE ZEILEN | HÆC DOMVS ODİT, AMAT, PVNIT, | CONSERVAT, HONORAT, | NEqVITIAM, PACEM, CRIMINA, | İVRA, PROBAT |

DİS HAVS HAST, LİEBT, STR[AFET] | BEHALTET, VERE[HR]ET | DİE SCHA[LC]KHEIT[, FRİ]D, LASTER, | RECHT [BİLIG]T. DİS HERET, | RATHS H[ER]RN: DEN REIM, NİE | LAST [AV]S DEM SİN KOMMEN | SO HINDERT İHR BESE VNDT | MEHRET DİE FROMMEN, | LESER NACH SEINEM TODT, | HIILF İHM AVS SEINER NOTH | SPRICH: GNADİG SEİ İHM GOTT.

Da der Text - trotz (auch heute verbreiteter) stereotyper negativer Vorstellungen von den verfilzten Strukturen und dem von Opportunismus bestimmten Agieren elitär besetzter politischer Gremien - die sehr persönliche Erfahrungsebene eines ,Insiders' (Ratsmitglieds) anspricht, ist man geneigt, die Autorenschaft eines Mietpoeten, die gegen Ende des 17. Jahrhunderts nicht ungewöhnlich gewesen wäre (vgl. Segebrecht 1977: 175-176, 183; Meid 1986: 36), ${ }^{2}$ als weniger wahrscheinlich zu

1 Die rekonstruierten Verse werden im Folgenden in eckigen Klammern beziffert.

2 Ein solches Engagement eines Berufsdichters durch die Hinterbliebenen des Verstorbenen folgte dem zeittypischen Motivationsmoment der ,Pflicht'. In St. Jakob gilt dies etwa für das Epitaph des Grafen de Souches, auf dem es heißt: „DİE GRAB SCHRIFFT HABEN İHM AUS WOHL 
betrachten und den Verstorbenen selbst dem Kreis möglicher Verfasser zuzuordnen. Als Apotheker ist ihm in jedem Fall ein Bildungsgrad zuzusprechen, der fundierte (klassische) Lateinkenntnisse, wie sie der Text ausweist, mit einschloss. Der kritische Ton der Verse, die weder Totenlob (laudatio), noch Trauerklage (lamentatio) oder Worte des Trostes (consolatio) enthalten, erhärtet diese Vermutung.

Auch liefert die Sprache des deutschen Bestandteils der Grabinschrift zumindest Indizien dafür: Denn - so erweist ein Vergleich mit dem Epitaph des Grafen de Souches - oberdeutsche Merkmale, die bei einem eingesessenen Brünner VerseSchmied zu erwarten gewesen wären, sind nur in reduziertem Ausmaß erkennbar. Dies könnte u. U. auf die westmitteldeutsche Herkunft des Verstorbenen (Mainz) zurückzuführen sein. Die wenigen Kennzeichen der in allen katholischen Gebieten des historischen deutschen Sprachraums verbreiteten oberdeutschen Schreib- und Literatursprache ${ }^{1}$ des 17 . und 18. Jahrhunderts sind folgende:

- die entrundete Wiedergabe von hdt. [ø:] als 〈e»:2 „HERET“ (,höret') [6], „BESE“ (,böse') [8],

- die e-Apokope im Nominativ Singular der Maskulina und im Nominativ/ Akkusativ Plural der Neutra: ${ }^{3}$ „[FRİ]D“ (,Friede'), „İAHR“ (,Jahrec) (biographische Angaben),

- die umlautlose Flexion der starken Verben mit Stammvokal $a$ in der 3. Person Singular: ${ }^{4}$ „BEHALTET“ (,behält') [5] sowie

- die adjektivisch gebrauchte Form „ZWO“ [2] des Zahlworts zwei im Feminin Plural. $^{5}$

VERMEINTEN PFLICHTEN, | SEINE KİNDS KİNDER DREŸ ANHERO LASSEN RICHTEN“ (zit. n. Blahak 2005: 33).

$1 \mathrm{Zu}$ dieser in den katholischen Gebieten des deutschen Reiches verbreiteten oberdeutschen Schriftnorm, die bis zur Mitte des 18. Jahrhunderts mit der von Martin Opitz propagierten ,meißnischen' (ostmitteldeutschen) Varietät konkurrierte, s. u. a. Breuer (1979: 44-48, 56; 1998; 2001: 90). Oberdeutsch dichtende Poeten der Barockzeit waren u. a. Jakob Balde (1604-1668), Sigmund von Birken (1626-1681), Albert Curtz (1600-1671), Hans Jakob Christoffel von Grimmelshausen (1622-1676), Georg Philipp Harsdörffer (1607-1658), Johannes Kuehn (16061675), Simon Rettenpacher (1634-1706), Abraham a Santa Clara (1644-1709) und Procopius von Templin (1609-1680) (vgl. Breuer 1979: 58-63, 80-85).

$2 \mathrm{Zu}$ schriftsprachlichen Üblichkeit der ö-Entrundung im (bairischen) Einzugsgebiet der oberdeutschen Schreib- und Literatursprache s. Tauber (1993: 72-75) und Reiffenstein (22003: 2050).

3 Zur schriftsprachlichen Üblichkeit der $e$-Apokope bei Substantiven im (bairischen) Einzugsgebiet der oberdeutschen Schreib- und Literatursprache s. Tauber (1993: 48-50) und Reiffenstein (22003: 2950).

4 Hierbei handelt es sich um ein im oberdeutschen und z. T. auch westmitteldeutschen (rheinpfälzischen) Raum regionalsprachlich verbreitetes Flexionsphänomen (vgl. Eichhoff 1978: 35; K. 123).

5 Sowohl im bairischen (vgl. Zehetner $\left.{ }^{4} 2014: 399\right)$ als auch im westmitteldeutschen Sprachraum (vgl. PWB 1993-1997: 1722-1724; SHW 2002-2010: 971) stellt zwo die adjektivisch gebrauchte Form des Zahlworts zwei im Feminin Plural dar. 
Dagegen fehlen folgende Merkmale, die für vergleichbare Grabinschriften aus dem bairischen Sprachraum ${ }^{1}$ typisch wären und die sich z. B. auch auf dem Grabepitaph des Grafen de Souches finden (vgl. Blahak 2005: 32-33, 37): ${ }^{2}$

- die unterbleibende Monophthongierung von mhd. uo (graphemisch als «ue fixiert): ${ }^{3}$ rueht statt „RVHT“ [2],

- e-Synkopen in den unbetonten Präfixen ge- und be-:4 gstrenge statt „GESTRENGE“ (biographische Angaben), bhaltet statt „BEHALTET“ [5],

- $n$-Flexionssuffixe bei maskulinen Definitartikeln und Possessivpronomen im Dativ Singular: 5 den statt „DEM“ [7], seinen statt „SEINNEM“ [9] sowie

\subsection{Struktur, Metrum und Aussage - ein Interpretationsversuch}

Mit der Grabinschrift wollte der Verfasser zweifellos einen Ausdruck bürgerlicher Gelehrsamkeit hinterlassen und zugleich, ironisch-pointiert verpackt, politische Systemkritik üben - an einem System, dem er selbst zu Lebzeiten angehörte und das er somit aus unmittelbarer Anschauung heraus kannte. Konstruiert wird ein Rahmen, der zwei rhetorische Sprechhaltungen miteinander verschränkt: Die Verse [1-2], in der $e r$-Erzählsituation eines anonymen Sprechers verfasst, fungieren als Einleitung zu einer folgenden Replik; sie stellen ein Mittel der Funktionalisierung dar, wie es auch bei wörtlicher Rede in Prosatexten und bei der Dramenrede verwendet wird. In dieser Replik geht die für den Barock typische Figur des ,sprechenden Toten ${ }^{6}{ }^{6}$ ausnahmsweise nicht einen beliebigen, des Weges kommenden Leser um Fürbitte an, sondern hinterlässt, so die Ankündigung, dem Rat der Stadt Brünn zugleich mit seinem ,letzten Seufzer' auch einen letzten Ratschlag; dieser entpuppt sich allerdings als ernüchternde Beschreibung eines (wenig schmeichelhaften) Ist-Zustandes- bzw. -Missstandes. Mit anderen Worten: Dem Brünner Rat wird ein Spiegel vorgehalten.

1 Ihm ist die Brünner Sprachinsel prinzipiell zuzuordnen (vgl. Beranek 1936: 277-278; Muzikant 2003: 97).

2 Einschränkend ist hier allerdings darauf hinzuweisen, dass Blahak (2014) am Beispiel von Grabinschriften des nördlichen Niederbayern feststellte, dass auch in anderen (,peripheren') katholischen deutschsprachigen Regionen in Grenzlage zum tschechischen Sprachgebiet - je nach Schichtzugehörigkeit des Verstorbenen/Textdichters - sprachliche Merkmale des Meißnischen in Grabschriften vorkommen können bzw. mit oberdeutschen Varianten konkurrieren.

3 Zu ihrer schriftsprachlichen Üblichkeit im (bairischen) Einzugsgebiet der oberdeutschen Schreibund Literatursprache s. Tauber (1993: 92-94) und Blahak (2014: 323).

$4 \mathrm{Zu}$ ihrer schriftsprachlichen Üblichkeit im (bairischen) Einzugsgebiet der oberdeutschen Schreibund Literatursprache s. Tauber (1993: 42-44) und Reiffenstein (2003: 2950).

$5 \mathrm{Zu}$ ihrer schriftsprachlichen Üblichkeit im (bairischen) Einzugsgebiet der oberdeutschen Schreibund Literatursprache s. Povejšil (1980: 86) und Blahak (2014: 325).

$6 \mathrm{Zu}$ dieser besonderen Darstellungsform des memento mori in der deutschen Barocklyrik s. u. a. Ingen (1966: 276-300) und Husenbeth (2007: 190-191). 
Hinsichtlich Opitz (1991) Forderung nach einem regelmäßigen Trochäus macht der Autor keinerlei Konzession an die zeitgenössischen Literaturnormen: Statt des üblichen sechshebigen, streng zwischen betonter und unbetonter Silbe alternierenden und durch eine zentrierte Zäsur gekennzeichneten Alexandriners $(\mathrm{u}-\mathrm{u}-\mathrm{u}-\| \mathrm{u}-\mathrm{u}-\mathrm{u}-(\mathrm{u}))$ wählt er für die deutschen Passagen seines Gedichtes [1-2, 5-8] das Metrum vierhebiger Knittelverse, die durch den Versfuß des Amphibrachys ( $\mathrm{u}-\mathrm{u}$ ) einen, rollenden' Rhythmus erhalten:

[1] $\mathrm{u}-\mathrm{u}|\mathrm{u}-\mathrm{u}| \mathrm{u}-\mathrm{u} \mid \mathrm{u}-\mathrm{u}$

[2] $u-u|u-u| u-u \mid u-u$

Mit dieser Abkehr vom Alexandriner verfolgte der Verfasser womöglich den Zweck, einen möglichst großen metrischen Kontrast zu den folgenden lateinischen Versen zu erzeugen: Denn kaum etabliert, werden die Knittelverse bereits in Vers [3] abrupt durch das sechshebige daktylische Versmaß zweier eingeschobener lateinischer Pentameter unterbrochen:

$$
\begin{aligned}
& \text { [3] - u u|-u u|-||-u u|- u u } \mid-\mathrm{u} \\
& {[4]-\mathrm{u} \mathrm{u}|--|-||-\mathrm{u}|-\mathrm{u}|-}
\end{aligned}
$$

Gleichzeitig mit dem unvermittelten Übergang zu diesem durch Zäsur geprägten Metrum erfolgen ein Sprach- und ein Sprecherwechsel. Hierdurch wird der folgende belehrende Sinnspruch gnomischen Typs ${ }^{1}$ dreifach als ,Zitat ${ }^{`}$ (hier einer gebildeten Persönlichkeit) gekennzeichnet.

Nun ist es der ,sprechende Tote', der - wenn auch ,zitiert' durch den anonymen Sprecher - auf Latein zu Wort kommt, in einem Sinnspruch in Form zweier nüchtern konstatierender Aussagesätze ohne Anrede oder Auslegung. Auch wenn zuvor gesagt wurde, es handle sich um einen Ratschlag des Verblichenen, erfolgen direkte Invokationen stets durch das Medium der, vortragenden Stimme', nicht durch das des ,sprechenden Toten': Mit den Worten „DİS HERET, | RATHS H[ER]RN“ [6-7] und „LESER [...] | HILLF İHM AVS SEINNER NOTH | SPRICH: GNADİG SEİ İHM GOTT" [9-11] wendet sich diese zudem an zwei voneinander unabhängige Adressaten: (1.) zunächst an den Rat der Stadt Brünn, dessen Mitglied der Verblichene selbst einst war, und (2.) abschließend an den homo viator, einen Wanderer, wie er seit der Antike von Grabinschriften bekannt ist, der (zufällig) in die allgemein menschliche Situation kommt, auf seinem (Lebens-)Weg kurz innezuhalten, um in Berichtform durch einen anonym bleibenden Sprecher, den die Inschrift vertritt, Informationen über eine wichtige Persönlichkeit zu empfangen (vgl. Segebrecht 1978: 435). Der Autor lässt dabei offen, wo das eigentliche Zitat

1 Zu dieser im 17. Jahrhundert häufigsten Erscheinungsform des Epigramms s. Weisz (1979: 80-98).

Brünner Hefte zu Deutsch als Fremdsprache • Jahrgang 9・Nummer $2 \bullet 2016$ 
endet. Ob die deutsche Übersetzung des ,Epigramms innerhalb eines Epigramms in Vers [5-6] auch noch dem Mund des Verstorbenen zuzuweisen ist oder der didaktischen Mitteilungsabsicht des anonymen Sprechers entspringt, bleibt offen, auch wenn durch die Rückkehr zum Knittelvers-Metrum und das ein Enjambement ankündigende, über die Übersetzung hinausführende „DİS HERET“ am Ende von Vers [6] Andeutungen in diese Richtung vorgenommen werden.

Denn muss der anonyme Sprecher den Räten der Stadt Brünn, einem Gremium, das per se nur aus vornehmen und gebildeten Bürgern bestehen sollte, die Botschaft eines lateinischen Sinnspruchs tatsächlich durch Übersetzung verständlich machen? Wird hier nicht womöglich mit Spott gerade auf in der Vergangenheit festgestellte eklatante Bildungslücken innerhalb der Ratsversammlung angespielt, deren Mitglieder im Ernstfall Schwierigkeiten hätten, die der humanistischen Gelehrsamkeit geläufige Form des lateinischen Pentameters metrisch wie sprachlich zu bewältigen? Der Inhalt der „ZWO [...] ZEILLEN“ [2] zumindest wirft unter Nutzung für den Barock typischer Stilmittel (Personifikation, ${ }^{1}$ asyndetische Häufung, ${ }^{2}$ Antithetik, ${ }^{3}$ Invokation, ${ }^{4}$ Enjambement ${ }^{5}$ ) ein höchst zwiespältiges Licht auf die politischen Entscheidungsprozesse im Brünner Rathaus: In diesem, so Vers [3], würden Entscheidungen nicht nach objektiven, dem Gemeinwohl dienenden Aspekten gefällt, sondern nach persönlichen, von Antipathie („DİS HAVS HAST“ [5]) und Sympathie („DİS HAVS [...] LİEBT“ [5]) bestimmten (und somit tendenziell rasch wechselnden) Allianzen. Je nach Konstellation und Stärke der jeweiligen ,Seilschaft käme es so - immer bei Fraglichkeit der Legitimität der Handlungsweise - zu Strafverfolgung („STR[AFET]“ [5]), Strafverschonung („BEHALTET“ [5]) oder sogar bevorzugter Behandlung („VERE[HR]ET“ [5]). Arglist, Gemeinheit („SCHA[LC]KHEIT“ [6]) und „LASTER“ [6] kämen demnach genauso zum Zuge wie die Garantie von Ruhe und Ordnung (,[FRİ]D“ [6]) und schließlich das eigentlich vom Stadtrat zu wahrende „RECHT“ [6].

Indem das Gedicht zwischen Vers [2] und [3] abrupt Erzählsituation/Sprecher, Sprache und Metrum wechselt, legt es eine literarische ,Falle' aus: Ein durch Vers [34] irritierter lesender Halbgebildeter wird sich durch die folgende Textübertragung

1 Personifiziert wird das Rathaus, das stellvertretend für den Brünner Stadtrat steht: „DİS HAVS HAST" [5].

2 In Vers [3-4] bzw. [5-6] bestimmen jeweils fünf Verben vier Akkusativ-Objekte (Substantive). Zur asyndetischen (unverbundenen) Häufung als beliebtes Stilmittel der Barockpoesie s. Braak (1979: 38).

3 Es stehen sich „ODİT“ und „AMAT“ [3] bzw. „HAST“ und „LIEBT“ [5], „CRIMIINA“ und „IVRA“ [4] bzw. „LASTER“ und „RECHT“ [6] sowie „BESE“ und „DİE FROMMEN“ [8] antithetisch gegenüber.

4 „DİS HERET, | RATHS H[ER]RN“ [6-7]. Zur Invokation, als im Barock „beliebtes Mittel, Häufungen nachdrücklicher und intensiver zu machen“, s. u. a. Braak (1979: 42).

5 „VERE[HR]ET | DIE SCHA[LC]KHEIT“ [5-6] und „DİS HERET, | RATHS H[ER]RN“ [6-7]); das Enjambement wird von den Barockpoetiken zur Auflockerung des Alexandrinerverses explizit empfohlen (vgl. Meid 1986: 54). 
zunächst kurzfristig erleichtert, beim Nachvollziehen des Gesagten jedoch sogleich verhöhnt fühlen, umso mehr als der anonyme Sprecher, gewissermaßen als ,Whistleblower, ${ }^{\text {' }}$ die Hinfälligkeit des städtischen Senats auch noch jedem beliebigen Jedermann vor Augen führt. Womöglich aus diesem Grund ergeht die Aufforderung an den ,homo viator' nicht (wie gemeinhin üblich) eingangs, sondern erst in der das Gedicht abschließenden Triole aus dreihebigen Versen.

Der Verblichene, als Apotheker des Lateinischen kundig, bleibt an diesem Spiel zumindest pro Forma unbeteiligt: Er kann sich gelassen auf die Position zurückziehen, nur zitiert worden zu sein. Die Anonymität des Sprechers befreit diesen „von der Subjektivität und Befangenheit vergänglicher Rede" (Segebrecht 1978: 435). Die Auslegung der Verse hat ein anderer, der er-Erzähler, in Vers [7-8] vorgenommen. Er selbst, der die kritischen Worte offenbar nur wohlgemeint im Privaten gesprochen hat, kann sich gegen den Vorwurf öffentlicher Nestbeschmutzung verwahren.

Erst in den beiden letzten Knittelversen löst sich das antithetische Spiel aus Mitwisserschaft und Teilhabe, Anklage und Distanz auf: Die beschriebenen Koordinaten des Brünner Stadtrats - so die Erkenntnis des Verstorbenen - sind offenbar nicht nur letztlich unabänderlich, sondern darüber hinaus charakteristisch ja: typologisch für alle von vielfältigen Interessen und machtpolitischen Ambitionen geprägten Gremien überhaupt. So blitzt die für die Barocklyrik typische Pointe am Ende des Gedichtes „wie ein Spiegel auf, in dem sich alles vorhergehende in unerwarteter und doch nicht ganz unvorbereiteter Weise reflektierend fängt" (Conrady 1962: 165): ${ }^{2}$ Der Einzelne könne diesem Nebeneinander von (subjektiv) ,rechtem' und ,unredlichem ' Agieren keinen grundsätzlichen Riegel vorschieben, doch solle er sich bei seinem persönlichen Handeln immer der benannten heterogenen Interessensstrukturen gewärtig sein, um so - gewissermaßen nach dem Leitsatz quidquid agis, prudenter agas et respice finem - dennoch zur zwar niemals idealen, doch bestmöglichen Lösung beizutragen: „DEN REIM, NİE | LAST [AV] S DEM SIN KOMMEN | SO HINDERT İHR BESE VNDT | MEHRET DİE FROMMEN“ [7-8].

\section{Zum Einsatz des Grabepitaphs im (hochschulischen) Literaturunterricht Deutsch als Fremdsprache}

\subsection{Vorüberlegungen zum didaktischen Potential der Grabinschrift}

Was macht diese Grabinschrift für das Fach Deutsch als Fremdsprache interessant? Die Praxiserfahrung im germanistischen Literaturunterricht an Hochschulen in Mittel- und Osteuropa zeigt, dass die Epoche des literarischen Barock vielen Lernern

1 Zum Begriff des Whistleblowings s. u. a. Ledergerber (2005) und Forst (2013).

2 Diese Ausrichtung auf ein überraschendes Ende "gehört zur Grundstruktur des Epigramms“ (Braak 1979: 41). 
fremdartig anmutet: Die zeitliche Ferne, der exemplarische Verweisungscharakter, das Theatralische und Formelhafte vieler Texte und die ungewohnte Bildsprache bereiten Schwierigkeiten. Hinzu kommt, dass man es mit einem aus heutiger Sicht nicht mehr zeitgemäßen Deutsch zu tun hat, das (wie im hier betrachteten Beispiel) womöglich auch noch mit anderen Sprachen (Latein, evtl. Französisch, Italienisch) versetzt ist. Das fremde Vokabular, eine ungewohnte Orthographie und Zeichensetzung verschlüsseln den Text zusätzlich. Verstärkt wird diese Textzugangsbarriere nicht zuletzt durch den Umstand, dass sich der Deutschunterricht an Gymnasien in Mittel- und Osteuropa weitgehend auf den Spracherwerb konzentriert, neben landeskundlichen Elementen also kaum Literaturunterricht beinhaltet, so dass in der Hochschulgermanistik in den seltensten Fällen von einem fundierten Vorwissen ausgegangen werden kann.

Aus fremdsprachendidaktischer Sicht lässt sich das Potential der hier abgedruckten poetischen Grabinschrift daher zunächst wie folgt zusammenfassen:

- Der Text ist kurz, er ,speichert' den Geist des Barock in epigrammatischer Form. Bei adäquater Wortschatzklärung lässt er sich von den Lernern problemlos erfassen und bleibt überschaubar.

- Er befindet sich in einem offen zugänglichen außerschulischen Lernraum, der den Alltag des Literaturunterrichts im Seminarraum durchbricht. Die gotische Hallenkirche und der im Halbdunkel platzierte ,geheimnisvolle' zerbrochene Stein, dessen ,Botschaft ${ }^{`}$ zu entschlüsseln ist, schaffen eine anregende Lernatmosphäre.

- Das Epitaph birgt einen deutschen Originaltext in einer nicht (mehr) deutschsprachigen Umgebung, eine in Stein gemeißelte authentische literarische Quelle im unmittelbaren Lebensumfeld des Lerners.

- Durch den Einbezug des Grabsteins als Textträger birgt die Grabinschrift zusätzliche Visualisierungseffekte, die stärker wirken als ein Gedicht auf Papier.

- Durch das Zusammenspiel von gotischer Hallenkirche, Grabmal, Text und Person ermöglicht das Epitaph einen fächerübergreifenden Unterricht, der Architektur, Literatur und (Sozial-)Geschichte aufs Engste miteinander verknüpft.

- Bei dem Bestatteten handelt es sich um eine Brünner Lokalgröße, was die Motivation zur Beschäftigung beim Lerner ansprechen dürfte, zumal er die ,eigene' Geschichte im Rahmen eines unkonventionellen Literaturunterrichts entdecken kann. Dies trägt zur Schulung der emotiven Kompetenz $z^{1}$ bei.

- Der durch einen Migrationshintergrund und Mehrsprachigkeit geprägte Werdegang des Brünner Ratsherrn ermöglicht auch die Schulung der interkulturellen Kompetenz (vgl. Kap. 4.2).

1 Zu den vom handlungs- und produktionsorientierten Literaturunterricht (vgl. Kap. 4.2) vermittelten Kompetenzen s. (auch im Folgenden) Haas (1997: 35-37). 
Das Epitaph bietet somit gutes Unterrichtsmaterial zur Verbindung der „beiden wichtigsten, einander ergänzenden und sich vielfach verschränkenden LernprozeßTypen [...] des entdeckenden Lernens einerseits und des nachvollziehendverstehenden Lernens andererseits" (Klafki ${ }^{4}$ 1994: 284).

\subsection{Der methodische Ansatz: ein handlungs- und produktionsorientierter, fächerübergreifender und interkultureller Literaturunterricht}

Als methodische Grundlage der Behandlung der Grabinschrift des Heinrich Nass im Rahmen der (Brünner) Hochschulgermanistik bietet sich zunächst der Ansatz des handlungs- und produktionsorientierten Literaturunterrichts an. Im Unterschied zu herkömmlichen hermeneutischen Paradigmen zielt dieser auf einen Lernprozess $a b$, in dem sich die Auseinandersetzung mit dem literarischen Text nicht nur auf Lesen und Analysieren beschränkt, sondern auch zu literarischästhetischen Ausdrucksformen führt: erzählendes und lyrisch gestaltendes Schreiben, bildliche oder dramatische Adaption, Vertonungen u. Ä. Dabei wird einerseits die Selbsttätigkeit der Lerner herausgefordert, ein ganzheitliches Handeln, das kognitive, sinnenhafte und affektive Zugänge verknüpft; andererseits werden operative Verfahren eingesetzt, welche die Lerner zu einem handelnden Umgang mit literarischen Texten auffordern. Ausgegangen wird dabei von der Prämisse, dass eigenes Tätigsein zu intensiveren Lernprozessen führt als das bloße Lesen, die Instruktion und das Unterrichtsgespräch. Durch das Bewusstmachen der ,Produziertheit' von Texten fördert das handlungs- und produktionsorientierte Verfahren die Textanalysekompetenz. Zugleich werden nicht-analytische Textbegegnungsformen als Verständnis- und Interpretationsleistung aufgefasst, die der Texterschließung dienen: eine Illustration, eine gestische Darstellung, eine erfundene Gegengeschichte können analoge, übertragene Verstehensweisen anstoßen und dokumentieren (vgl. Spinner ${ }^{5} 2010$ : 247, 252-253).

Der gewählte Ansatz sollte zudem fächerübergreifend zum Einsatz kommen; die Grenzen zwischen der Germanistik und anderen Fächern, die sich mit dem Barockzeitalter beschäftigen (u. a. Kunstgeschichte, Kulturwissenschaft, Geschichte und Soziologie), sollten überschritten werden. Zudem bietet es sich, wie Kap. 3.1 plausibel gemacht hat, auch an, die ,intra-philologische' Grenze zwischen Literaturund Sprachwissenschaft aufzuheben. ${ }^{1}$ Grundsätzlich fördern fächerübergreifende Lernkonzepte eine multiperspektivische Wahrnehmung der Wirklichkeit und

1 Dies gilt nicht zuletzt deswegen, weil die Barockliteratur auch immer im Zentrum der Bemühungen um die Pflege der deutschen Sprache (Sprachgesellschaften) und die Schaffung einer einheitlichen Norm der deutschen Hochsprache (Opitz) stand. Henne (1966: 15) bezeichnet die frühe Barockliteratur als vorläufige „hochsprachliche Übung“. Dass sie in Manchem danebengreife, zeige zwar das allgemeine Bestreben an, hinsichtlich eines Hochdeutschen überein zu kommen. Doch stellten die damaligen Postulate noch eher hochgesteckte Ziele als angehende Verwirklichung dar. 
damit einhergehend die Entwicklung übergreifender Einsichten, Fähigkeiten, Arbeitsmethoden und Lernstrategien, welche die Entwicklunggemeinsamer Klärungsund Lösungsstrategien begünstigen (vgl. Schindler ${ }^{5} 2010: 280$ ). Formen des offenen Unterrichts, besonders die Projektarbeit, stellen die in der didaktischen Literatur hauptsächlich empfohlenen Konzepte für fächerübergreifenden Unterricht dar (vgl. Leubner, Saupe und Richter 2010: 215), wobei komplementäre, konzentrische, kontrastive/dialogische oder reflexive Verhältnisse der zusammenwirkenden Fächer vorgeschlagen werden (vgl. Huber 1997: 65).

Schließlich liegt es auch nahe, pädagogische und fachdidaktische Elemente des interkulturellen Lernens zu berücksichtigen, bei der „Umstrukturierung selbstorganisierter Ich- und Weltentwürfe anhand einer Neuinterpretation kultureller Repräsentationen, die durch den Rückgriff auf den Verstehenshorizont des anderen [...] initialisiert wird" (Dawidowski 2006: 25). Der durch Mehrsprachigkeit geprägte ,Projektgegenstand', das Grabepitaph des Heinrich Nass, der, aus dem westmitteldeutschen Sprachraum (Mainz) stammend, eine deutsch-lateinische Grabinschrift in einem (ober-)deutsch-tschechisch(-lateinisch)-sprachigen Umfeld hinterließ, ist prädestiniert für das übergreifende Lernziel, das der interkulturelle Literaturunterricht verfolgt: die „Entwicklung der Individualität und der Fähigkeit zur sozialen Interaktion in der polykulturell geprägten Gesellschaft durch Teilnahme am Handlungsfeld Literatur" (Leubner, Saupe und Richter 2010: 221).

\subsection{Unterrichtsvorschläge}

Nach einer ersten theoretischen Erarbeitung zentraler Merkmale des Barockgedichts an einem geläufigen Beispiel (z. B. Gryphius' Vanitas-Sonett) und der Klärung des historisch-politischen und gesellschaftlich-kulturellen Hintergrundes der Barockzeit auf europäischer und regionaler Ebene (z. B. in Form von Referaten) sind folgende Arbeitsaufträge an die Lerner denkbar: Zum Einstieg könnte man - als „stummen Bildimpuls" (Abraham und Kepser 2005: 178) - Fotografien des (bescheidenen) Grabmals des Heinrich Nass und des (prunkvollen) Grabmals des Grafen de Souches in der Brünner St. Jakobskirche kontrastieren, um das Barock als Zeitalter der Gegensätze und Spannungen (vgl. die Schlagwörter vanitas vs. carpe diem bzw. die der Barocklyrik geläufige Antithetik) bildlich vor Augen zu führen.

In der Textbegegnungsphase sollten die Lerner in einem weiteren Schritt den Epitaphtext soweit wie möglich transkribieren, mit Hilfe von Wörterbüchern den Wortschatz klären und den Textverlust (unter Berücksichtigung der lateinischen Verse) rekonstruieren (vgl. Kap. 3.1). Weiter können sie - zur leichteren Lesbarkeit und zur weiteren Bearbeitung - Satzzeichen nach heutigen Regeln einfügen, gegebenenfalls auch die Orthographie an die heutige Norm anpassen. Ferner können nach Bestimmung des Metrums des Knittelverses und des Pentameters (der vom Lehrer erläutert, aber auch durch die Lerner in eigener Recherchearbeit identifiziert 
werden kann) Betonungszeichen und Zäsuren eingefügt werden (Schulung der literarischen Kompetenz). In dieser Texterfassungsphase wäre auch eine Übertragung der Grabinschrift in ein modernes Neuhochdeutsch bzw. in die Muttersprache der Lerner (Gruppenarbeit) zur Erleichterung der folgenden Arbeitsschritte denkbar.

In der Texterarbeitungsphase kann wie folgt vorgegangen werden: Nach der Sicherung des Textverständnisses können in Lernergruppen die inhaltliche Gliederung des Gedichtes (Rahmen, Replik) und die Sprechersituation herausgearbeitet werden sowie ein Gattungsbestimmungsversuch erfolgen; schließlich wird die Herausarbeitung der augenfälligsten dichterischen Stilmittel des Barock (vgl. Kap. 3.2) keine Schwierigkeiten mehr bereiten (Schulung der ästhetischen Kompetenz).

Eine folgende, in Gruppen zu bewältigende Aufgabe wäre die Identifizierung der im biographischen Informationstext des Grabepitaphs genannten Orte (Brünn, Mainz). Darauf aufbauend können die Lerner einerseits auf einer historischen Mitteleuropakarte bzw. einer Dialekt-Karte des historischen deutschen Sprachraums ein Itinerar des Apothekers entwerfen und den Migrationsweg des Protagonisten, die Vielzahl der dabei durchquerter Territorien (mit ihren unterschiedlichen konfessionellen und staatsrechtlichen Parametern) bzw. deutschen und tschechischen Sprachlandschaften visualisieren, um die interkulturelle Dimension dieses Ortswechsels nachzuvollziehen. Auf der anderen Seite bieten sich Rechercheaufgaben zu den im Text explizit genannten (Rathaus, Apotheke Zum Roten Krebsen) und implizit anwesenden Lokalitäten (St. Jakob) an: Mithilfe historischer Darstellungen ${ }^{1}$ gilt es, das Hintergrundwissen zu diesen mit objektiven Fakten zu verfestigen und dieses mit den Aussagen des Epitaphs zu vergleichen, um im Anschluss die ,Objektivität‘ (d. h. den Rollencharakter), die dem Sprecher/Autor des Epitaphs beigemessen werden kann, zu bewerten (Schulung der kritischen Kompetenz).

Je nach Kenntnisstand der Lerner kann sich auch eine Bewertung der literarisch-sprachlichen Güte des Textes (etwa im Vergleich mit dem u. U. bereits bekannten Gryphius-Sonett) anschließen. Eine zusammenfassende Diskussion zur Beantwortung der Frage Was ist, barock' an diesem Text? kann diese Arbeitsphase (Textsicherungs- bzw. Übertragungsphase) abschließen. Dabei sollte die Aktualität der Systemkritik des barocken Ratsherrn mit seinem Insider-Blick in politische Entscheidungsgremien nicht außer Acht gelassen werden - sie kann im Unterricht zur Diskussion über politische Skandale der Gegenwart (z. B. über die Korruptionsdebatte um die FIFA oder die Aufdeckung von Steuerhinterziehungsvergehen prominenter Politiker in Millionenhöhe im Rahmen der Panama- und Bahama-Papers) oder den Akt des Whistleblowings (vgl. den Fall Edward Snowden) führen (Schulung der Projektionskompetenz).

1 U. a. Bretholz (1901; 1938), Kožíšek (1928), Reutter und Altrichter (1942), Ludwig und Chlumecky (1972), Waldner (1980), Válka (1995) und Čapka (2001). 
Je nach der zur Verfügung stehenden Zeit sollte man nach Möglichkeit auf diesen fachlich-interpretatorischen Teil noch eine projektorientierte Produktionsphase zur Schulung der kreativen Kompetenz folgen lassen. Einige Ideen hierfür wären u. a.:

- Spiel mit dem medialen Ort des Textes durch die Wahl eines anderen Mediums, etwa

- eines TV-Features, in dem der Tod des Ratsherrn (im Stil einer heutigen Sensationsreportage über einen Prominenten) gemeldet wird, ein Nachruf mit seinem Werdegang erfolgt oder über den Skandal, den die Offenlegung der Machenschaften im Brünner Rat in der Öffentlichkeit verursacht hat, berichtet wird,

- einer Sterbeannonce in einer Tageszeitung oder

- einer Wahlkampfrede im Vorfeld der Neubesetzung des Brünner Stadtrats;

- Erstellung einer tschechischen Version der bereits auf Deutsch und Latein existierenden mahnenden Verse [3-4] bzw. [5-6] des Apothekers, die ebenfalls ein eigenes Metrum erhält;

- Umformung des Epitaphtextes zu einem Rap/Hip-Hop-Song nach Vorbildern aus der aktuellen Musikszene. ${ }^{1}$

\section{Fazit}

Das Beispiel des Grabepitaphs des Brünner Apothekers und Ratsherrn Heinrich Nass zeigt exemplarisch, dass auch an der (vermeintlichen) ,Peripherie‘ des historischen deutschen Sprachraums eine Barockliteratur von Format verfertigt wurde, die in ihren Möglichkeiten des kunstvollen literarischen Spiels den ,binnendeutschen' Vertretern des Barocks offenbar in nichts nachstand. Zugleich macht es die regional gebundene (oberdeutsche) wie ,europäische` Dimension barocker Casualcarmina am Rande des deutschen Sprachgebietes fassbar. Nicht zuletzt lenkt es den Blick auf eine noch wenig erforschte literarische Textsorte des Deutschen in den böhmischen Ländern und lädt dazu ein, diese im Rahmen eines handlungs- und produktionsorientierten, fächerübergreifenden und interkulturellen Literaturunterrichts einzusetzen, der sich (auch) an außerschulischen Lernorten abspielt, die Teil des Lerner-Lebensumfeldes sind.

1 Hierdurch würde in das bereits skizzierte Konzept des fächerübergreifenden und projektorientierten Unterrichts auch noch die Musik einbezogen. 


\section{Abbildungsverzeichnis}

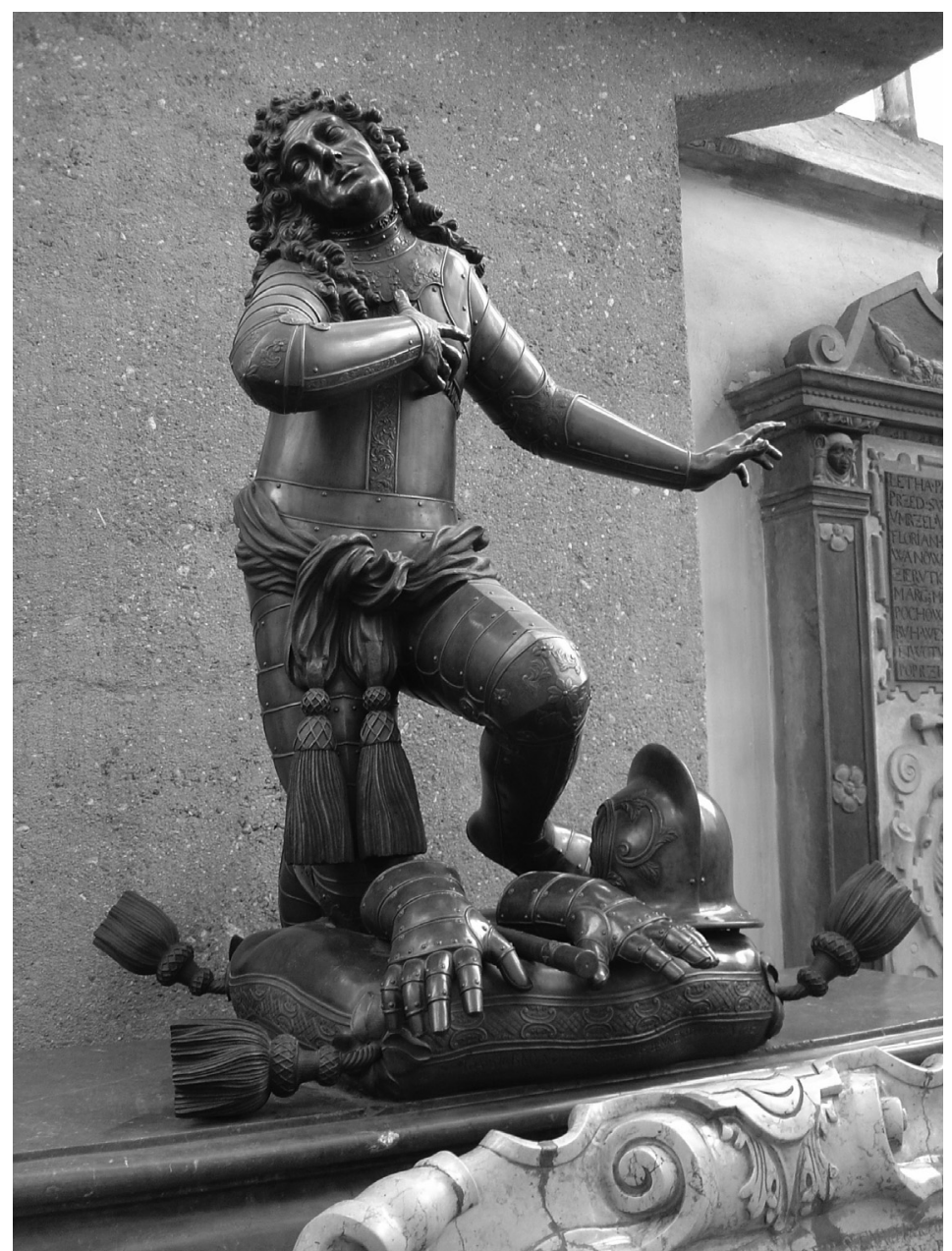

Abb. 1: Das Grabmal des kaiserlichen Feldmarschalls Louis Rattuit de Souches von Johannes Sigismund Kerker in St. Jakob/Brünn (Foto: B. Blahak, 2012) 


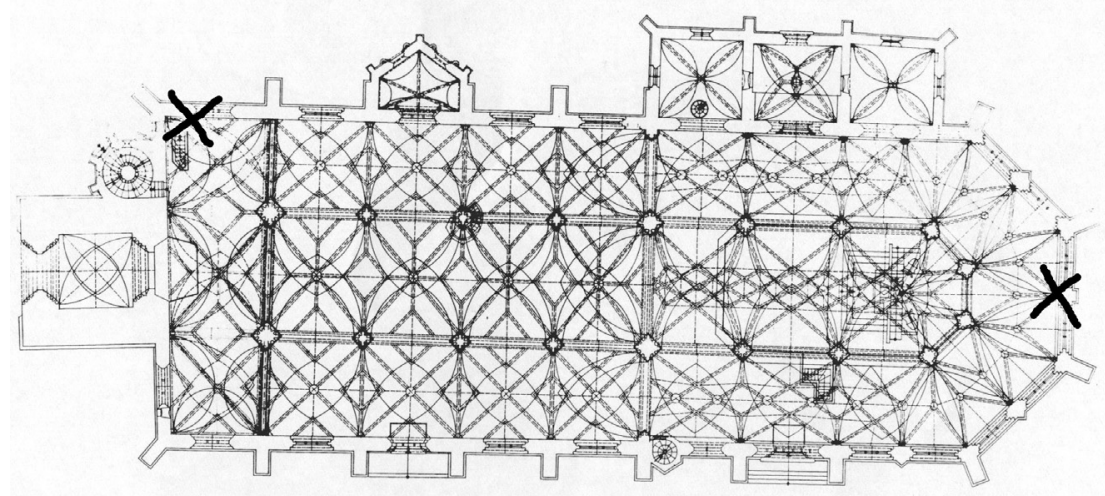

Abb. 2: Die Standorte der Grabepitaphe des Heinrich Nass (links) und des Grafen de Souches (rechts) in St. Jakob/Brünn (Slabá und Rutrlová www)

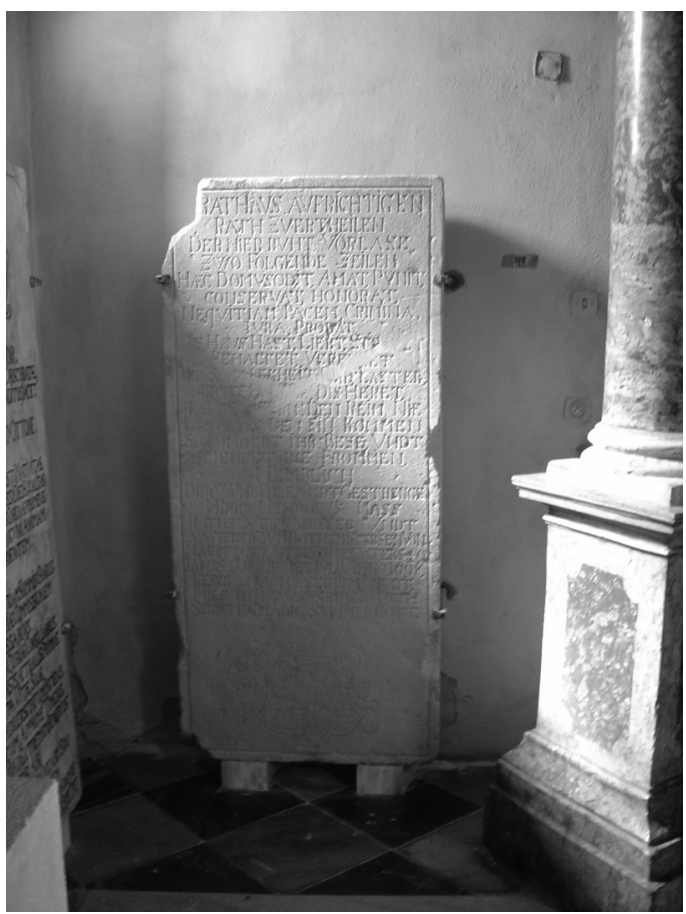

Abb. 3: Das Grabmal des Brünner Ratsherrn und Apothekers Heinrich Nass in St. Jakob/Brünn (Foto: B. Blahak, 2012) 


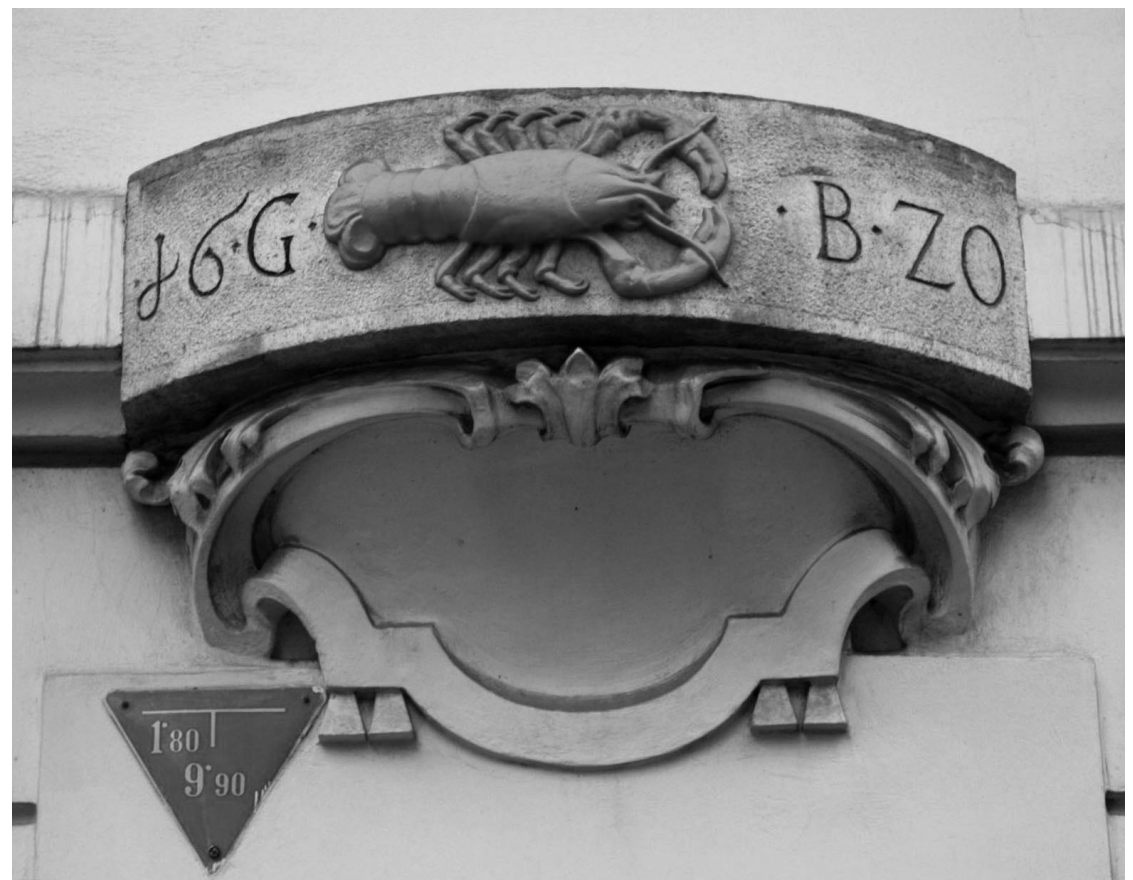

Abb. 4: Hauszeichen, Apotheke Zum roten Krebsen/Brünn (Foto: B. Blahak, 2016)

\section{Literaturverzeichnis}

Abraham, Ulf / Kepser, Matthis (2005): Literaturdidaktik Deutsch. Eine Einführung. Berlin, Erich Schmidt.

Beck, Adolf (1965): Über ein Gedicht von Georg Rudolf Weckherlin und seinen formtypologischen Bereich. In: Schillemeit, Jost (Hg.): Deutsche Lyrik von Weckherlin bis Benn (= Interpretationen 1). Frankfurt a. Main, Fischer. S. 11-18.

Beranek, Franz L. (1936): Die Mundart von Südmähren (Lautlehre) (= Beiträge zur Kenntnis Sudetendeutscher Mundarten 7). Reichenberg, Anstalt für Sudetendeutsche Heimatforschung.

Blahak, Boris (2005): „RED İTZT O PRAGER SCHLACHT // WIE DIESER LÖW GEKÄMPFT“. Das Grabepitaph des Grafen de Souches in Brünn. Ein projektorientierter Ansatz zum Barockgedicht im Literaturunterricht $\mathrm{DaF}$ unter Berücksichtigung des historischen Lernerumfeldes im außerschulischen Lernraum. - In: Váňa, Pavel (Hg.): Brünner Hefte für Germanistik (= Sborník prací pedagogické fakulty Masarykovy univerzity v Brně 184). Brno, Masarykova univerzita. S. 31-42.

Blahak, Boris (2014): „Sich Wer Wir Seindt Gewesen.“ Mundartnähe und -ferne auf poetischen Grabepitaphen der Barockzeit zwischen Donau und Böhmerwald. In: Frieben, Elisabeth / Kanz, Ulrich / Neuber, Barbara / Zehetner, Ludwig (Hg.): Dialekt und Religion. Beiträge zum 5. Dialektologischen Symposium im Bayerischen Wald, Walderbach, Juni 2012 (= Regensburger Dialektforum 20). Regensburg, Vulpes. S. 305-334. 
Braak, Ivo (1979): Gattungsgeschichte deutschsprachiger Dichtung in Stichworten, Teil II b. Lyrik. Vom Barock bis zur Romantik. Kiel, Ferdinand Hirt.

Bretholz, Berthold (1901): Die Pfarrkirche St. Jakob in Brünn. Brünn, Gemeinderath der Landeshauptstadt Brünn.

Bretholz, Berthold (1938): Brünn. Geschichte und Kultur. Aus dem Nachlass herausgegeben von der Deutschen Gesellschaft für Wissenschaft und Kunst in Brünn. Brünn, Rohrer.

Breuer, Dieter (1979): Oberdeutsche Literatur 1565-1650. Deutsche Literaturgeschichte und Territorialgeschichte in frühabsolutistischer Zeit $(=$ Zeitschrift für bayerische Landesgeschichte. Beiheft 11. Reihe B). München, Beck.

Breuer, Dieter (1998): Raumbildungen in der deutschen Literaturgeschichte der frühen Neuzeit als Folge der Konfessionalisierung. In: Zeitschrift für Deutsche Philologie 117 (Sonderheft „Regionale Sprachgeschichte“). S. 180-191.

Breuer, Dieter (2001): Die protestantische Normierung des deutschen Literaturkanons in der Frühen Neuzeit. In: Haupt, Heinz-Gerhard / Langewiesche, Dieter (Hg.): Nation und Religion in der deutschen Geschichte. Frankfurt a. Main [u. a.], Campus-Verlag. S. 84-104.

Čapka, František (2001): Dějiny Moravy v datech. Brno, Akademické Nakladatelství CERM.

Conrady, Karl Otto (1962): Lateinische Dichtungstradition und deutsche Lyrik des 17. Jahrhunderts (= Bonner Arbeiten zur deutschen Literatur 4). Bonn, H. Bouvier.

Dawidowski, Christian (2006): Theoretische Entwürfe zur interkulturellen Literaturdidaktik. Zur Verbindung pädagogischer und deutschdidaktischer Interkulturalitätskonzepte. In: Dawidowski, Christian / Wrobel, Dieter (Hg.): Interkultureller Literaturunterricht. Konzepte - Modelle - Perspektiven. Baltmannsweiler, Schneider-Verlag Hohengehren. S. $169-190$.

Dostál, E. (1928): Die Kunstdenkmäler Brünns. In: Kožíšek, Alois V. (Hg.): Die Landeshauptstadt Brünn. Prag, Emil Šolc. S. 46-59.

Eichhoff, Jürgen (1978): Wortatlas der deutschen Umgangssprache. Bd. 2. Bern [u. a.], A. Francke / K. G. Saur.

Forst, Gerrit (2013): Whistleblowing im internationalen Vergleich - Was kann Deutschland von seinen Nachbarn lernen? In: Europäische Zeitschrift für Arbeitsrecht 2013/6/1. S. 37-82.

Haas, Gerhard (1997): Handlungs- und produktionsorientierter Literaturunterricht. Theorie und Praxis eines „anderen“ Literaturunterrichts für die Primar-und Sekundarstufe. SeelzeVelber, Kallmeyer.

Henne, Helmut (1966): Hochsprache und Mundart im schlesischen Barock. Studien zum literarischen Wortschatz in der ersten Hälfte des 17. Jahrhunderts (= Mitteldeutsche Forschungen 44). Köln, Böhlau.

Hoffmannswaldau, Christian Hoffmann von (1662): Hundert Grab-Schrifften. Mit willen in zwey Theile (also daß das eine meist Historische daß ander aber ergötzliche Materien vorstelle) getheilet Vnd Der untadelhafften Welt zum Zeitvertreib zugeschicket. o. O., o. V.

Huber, L. (1997): Organisationsformen fächerübergreifenden Unterrichts. In: Ansätze zum fächerübergreifenden Unterricht. Hrsg. vom Landesinstitut für Schule und Weiterbildung. Bönen, Kettler. S. 53-75.

Husenbeth, Helmut (2007): „Es ist ein Schnitter, heißt: der Todt“. Sterben, Tod und Auferstehung im geistlichen Lied des 17. Jahrhunderts (= Koblenz-Landauer Studien zu Geistes-, Kulturund Bildungswissenschaften 2). Trier, Wissenschaftlicher Verlag.

Ingen, Ferdinand van (1966): Vanitas und memento mori in der deutschen Barocklyrik. Groningen, J. B. Walters.

Klafki, Wolfgang ( $\left.{ }^{4} 1994\right)$ : Neue Studien zur Bildungstheorie und Didaktik. Zeitgemäße Allgemeinbildung und kritisch-konstruktive Didaktik. Weiheim / Basel, Beltz. 
Kožíšek, Alois V. (Hg.) (1928): Die Landeshauptstadt Brünn. Prag, Emil Šolc.

Kühlmann, Wilhelm (1982): Sterben als heroischer Akt. Zu Paul Flemings Grabschrifft. In: Meid, Volker (Hg.): Gedichte und ihre Interpretationen. Bd. 1. Stuttgart, Reclam. S. $168-175$.

Ledergerber, Zora (2005): Whistleblowing unter dem Aspekt der Korruptionsbekämpfung. Bern, Stämpfli.

Leubner, Martin / Saupe, Anja / Richter, Matthias (2010): Literaturdidaktik. Berlin, Akademie Verlag.

Ludwig, Georg / Chlumecky, Peter von (1972): Des Rathsherrn und Apothekers Georg Ludwig Chronik von Brünn. 1555-1604 (Reprint der Ausgabe Brünn 1859). Wien, Geyer.

Meid, Volker (1986): Barocklyrik. Stuttgart, Metzler.

Muzikant, Mojmír (2003): Tschechische Lehnwörter im Deutschen Südmährens. In: Greule, Albrecht / Nekula, Marek (Hg.): Deutsche und tschechische Dialekte im Kontakt. Wien, Praesens. S. 95-100.

Opitz, Martin (1991): Buch von der Deutschen Poeterey (1624). Bibliographisch ergänzte Ausgabe. Stuttgart, Reclam.

Povejšil, Jaromír (1980): Das Prager Deutsch des 17. und 18. Jahrhunderts. Ein Beitrag zur Geschichte der deutschen Schriftsprache. Hamburg, Buske.

PWB (1993-1997): Pfälzisches Wörterbuch. Hrsg. von Ernst Christmann und Julius Krämer. Bd. 6. Wiesbaden, Steiner.

Reiffenstein, Ingo (2003): Aspekte einer bayerischen Sprachgeschichte seit der beginnenden Neuzeit. In: Besch, Werner / Betten, Anne / Reichmann, Oskar / Sonderegger, Stefan (Hg.): Sprachgeschichte. Ein Handbuch zur Geschichte der deutschen Sprache und ihrer Erforschung. Bd. 3. Berlin [u. a.], de Gruyter. S. 2942-2971.

Reutter, Hans / Altrichter, Anton (1942): Drei Jahrhunderte Brünner Bürgertum 1559-1843. München, Rohrer.

Schindler, Frank (52010): Verbundsysteme. Integrativer Deutschunterricht und fächerübergreifendes Lernen. In: Bogdal, Klaus-Michael / Korte, Hermann (Hg.): Grundzüge der Literaturdidaktik. München, Deutscher Taschenbuch Verlag. S. 272-285.

Segebrecht, Wulf (1977): Das Gelegenheitsgedicht. Ein Beitrag zur Geschichte und Poetik der deutschen Lyrik. Stuttgart, Metzler.

Segebrecht, Wulf (1978): Steh, Leser, still! Prolegomena zu einer situationsbezogenen Poetik der Lyrik, entwickelt am Beispiel von poetischen Grabschriften und Grabschriftenvorschlägen in Leichencarmina des 17. und 18. Jahrhunderts. In: Deutsche Vierteljahresschrift für Literaturwissenschaft und Geistesgeschichte 1978/52. S. 430-468.

Segebrecht, Wulf (1981): Poetische Grabschriften des 17. Jhs. Als literarische Zeugnisse des barocken Lebensgefühls. In: Literatur für Leser 1981/1. S. 1-17.

Segebrecht, Wulf (1987): Über poetische Grabschriften. In: Segebrecht, Wulf (Hg.): Poetische Grabschriften. Frankfurt a. Main, Insel. S. 129-136.

SHW (2002): Südhessisches Wörterbuch. Hrsg. von Friedrich Maurer und Rudolf Mulch. Bd. 6. Marburg, N. G. Elwert.

Slabá, Lucie / Rutrlová, Jana (www): Kostel sv. Jakuba, Brno. URL <http://www.spsstavbrno. cz/dokumenty/Socrates/html/cz/stavby/kostel_sv_jakuba_cz.html> [Zugriff am 30.9.2016].

Spinner, Kaspar H. (52010): Handlungs- und produktionsorientierter Literaturunterricht. In: Bogdal, Klaus-Michael / Korte, Hermann (Hg.): Grundzüge der Literaturdidaktik. München, Deutscher Taschenbuch Verlag. S. 247-257.

Tauber, Walter (1993): Mundart und Schriftsprache in Bayern (1450-1800). Untersuchungen zur Sprachnorm und Sprachnormierung im Frühneuhochdeutschen. Berlin / New York, de Gruyter. 
Válka, Josef (1995): Dějiny Moravy. Díl 2. Morava. Reformace, renesance a baroka. Brno, Muzejní a vlastivědná společnost.

Waldner, Wolfgang F. (1980): Das deutsche Brünn. Ein Beitrag zur kulturhistorischen Entwicklung der Landeshauptstadt Mährens. Erweiterte schriftliche Fassung eines Vortrages, gehalten in Nürnberg am 12. Okt. 1980. Nürnberg, Bundesverband der „BRUNA“.

Weisz, Jutta (1979): Das deutsche Epigramm des 17. Jahrhunderts (Germanistische Abhandlungen 49). Stuttgart: Metzler.

Zehetner, Ludwig ( $\left.{ }^{4} 2014\right)$ : Bairisches Deutsch. Lexikon der deutschen Sprache in Altbayern. Regensburg, Vulpes.

Dr. phil. Boris Blahak, M.A.

Institut für Germanistik

Deutsch als Fremdsprachenphilologie

Universität Regensburg

Universitätsstraße 31

93053 Regensburg

boris.blahak@ur.de

Katedra germanistiky a slavistiky

Filozofická fakulta

Západočeské university v Plzni

Riegrova 11

30614 Plzeň

bblahak@kgs.zcu.cz 